\title{
Bilobar congenital lobar emphysema in a child: how to approach it?
}

\author{
Krishna Kumar Govindarajan \\ Jawaharlal Institute of Postgraduate Medical Education and Research, Pondicherry, India
}

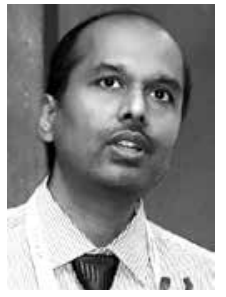

Kardiochir Torakochir Pol 2020; 17 (4): 208-209

A 1-month-old, term male child, with a birth weight of $3.6 \mathrm{~kg}$, presented with sudden onset respiratory distress. There was no accidental foreign body inhalation or oil instillation. Plain X-ray chest showed bilateral emphysematous involvement (left upper lobe and right middle lobe), left more severe than the right as evidenced by the mediastinal shift and lung herniation (Figure $1 \mathrm{~A}$ ). The child was resuscitated and supported with oxygen. In view of worsening respiratory distress, the child was intubated and ventilated. Intravenous antibiotics were commenced to treat the associated pneumonia. Over a period of 1 week, the ventilator settings could not be weaned, pointing to the surgical lesion as the obvious cause. Chest computed tomography (CT) was consistent with bilobar emphysematous involvement without any obvious extraluminal bronchial compression. As the left upper lobe was acting as a space occupying lesion causing lung herniation to the opposite side, left upper lobectomy was planned.

Written informed high risk consent was obtained from the parents. In view of the technically demanding proce- dure and the urgency of the situation, open lobectomy was preferred over video assisted thoracoscopic surgery. Lobectomy was performed by a conventional left posterolateral thoracotomy via the $4^{\text {th }}$ intercostal space (Figure 2). As single lung ventilation in a neonate was deemed unwarranted, a standard endotracheal tube was used. The pulmonary vein and artery to the lobe were identified and ligated individually. The upper lobe bronchial stump was oversewn with 5/0 Prolene sutures. The left lower lobe expanded well after removal of the upper lobe. After an uneventful post-operative course, the child was extubated on day 1. He continued to remain stable without respiratory distress and was discharged home on post-operative day 5 . As the child was well, the right middle lobectomy was deferred and observation advised. However, the child developed distress after 10 days and was re-admitted. The right middle lobe was prominently emphysematous on the plain X-ray chest, with satisfactory left lower lobe expansion filling the hemithorax. The child required intubation and ventilation
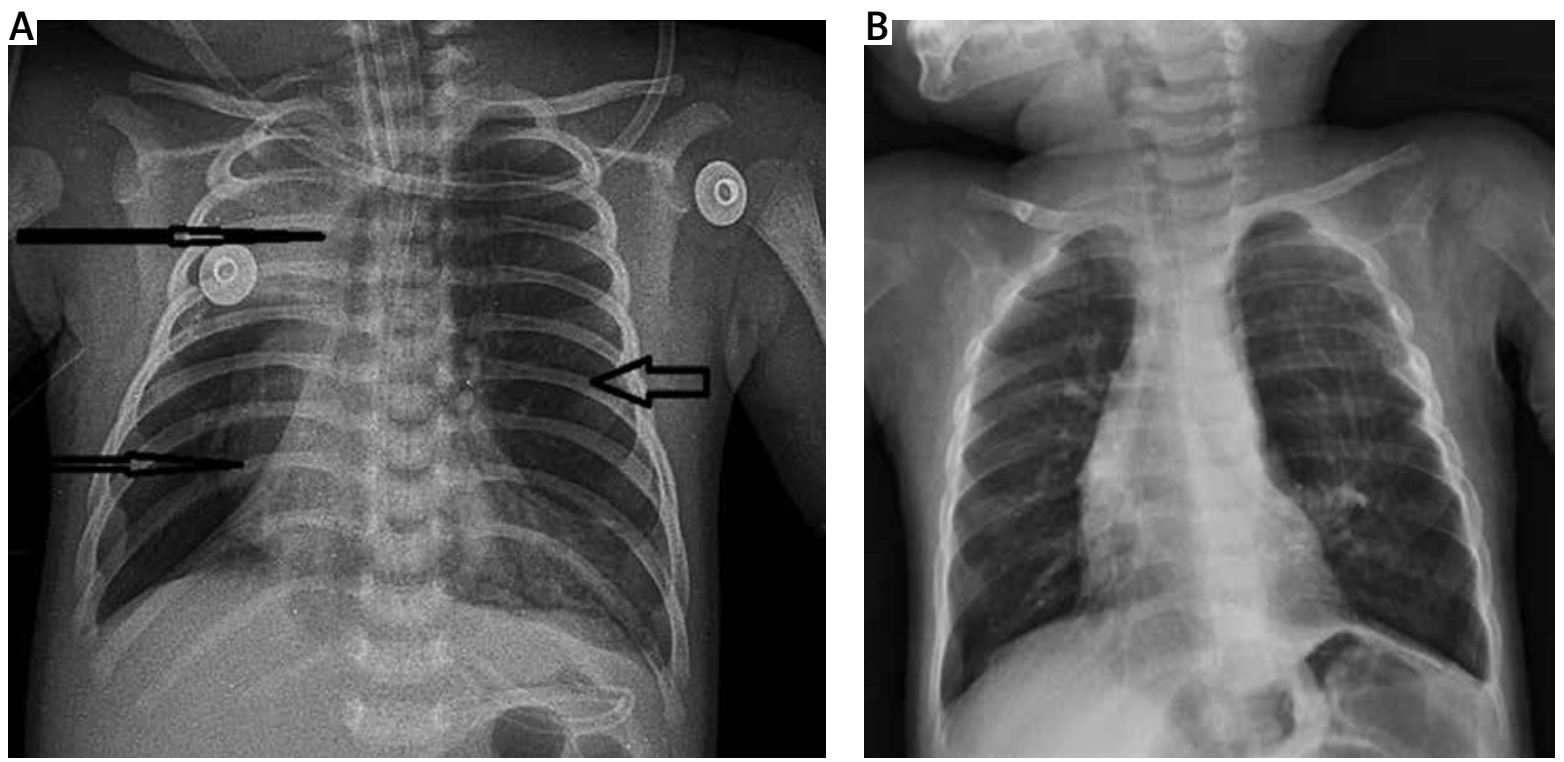

Figure 1. A - Plain chest X-ray showing right middle lobe emphysema (short arrow) with collapsed lower lobe, left upper lobe emphysema (arrow) causing mediastinal shift and lung herniation (long arrow). B - Plain chest X-ray after bilobectomy

Address for correspondence: Krishna Kumar Govindarajan, Jawaharlal Institute of Postgraduate Medical Education and Research, Pondicherry, India, e-mail: kkpeds@gmail.com

Received: 14.07.2020, accepted: 27.09.2020. 
for the distress. Following a week of stabilization and intravenous antibiotics, the child was taken up for surgery. After written informed high risk consent, he underwent a right middle lobectomy by standard right posterolateral thoracotomy. The vessels and the bronchial stump to the middle lobe were identified and secured separately.

The child's distress settled in the post-operative period, enabling extubation on day 2. On continued observation, he was well on room air and was subsequently sent home by day 6 . The bilateral thoracotomy scars were healthy with good lung expansion on plain X-ray chest (Figure $1 \mathrm{~B}$ ). He has completed 1-year follow-up and remained symptom free with satisfactory weight gain and age appropriate milestones. Histopathology of both the excised lobes confirmed the diagnosis of congenital lobar emphysema (CLE).

With an incidence of 1 in 20,000 to 1 in 30,000 live births, congenital cystic lung lesions are rare in children [1]. The majority of them are identified on the antenatal scans and add to the dilemma of management in the postnatal period with the presentation of an asymptomatic neonate to the outpatient clinic. Causes for congenital cystic lung lesions in children include congenital cystic adenomatoid malformation, CLE, lobar sequestration, foregut duplication, and lung cyst. Among these, all except CLE usually get detected in the anomaly scan in the second trimester [2]. Utility of magnetic resonance imaging in the antenatal diagnosis of CLE has been documented [3]. Children with CLE present in the first week of life in about $50 \%$ of cases with the rest becoming symptomatic subsequently in the next 6 months of age. Although life-threatening severe respiratory distress is noted in children with CLE, instances of CLE without distress have been documented, which may require observation [1].

Primarily, the dysplasia or hypoplasia of the bronchial cartilage is the etiological factor in the causation of CLE, resulting in ball valve like air trapping, making the involved lobe emphysematous with compression of the adjacent lobe causing respiratory distress [4].

Rudhe syndrome, proposed to be due to left to right cardiac shunts due to VSD/PDA resulting in right middle lobe CLE, underlines the role for echocardiography [5]. Where possible, bronchoscopy to rule out mucus plugging and echocardiography to look for aberrant vessels causing extraluminal blockage of bronchus form part of the preoperative work-up. CLE is a disease of the upper lobes, as is evident in the order of occurrence (left upper lobe $>$ right middle lobe > right upper lobe), with less than $1 \%$ lower lobe involvement. Male predominance is identified in bilateral CLE. Our patient was also a boy, in line with the literature [6].

Bilateral involvement may escape medical attention and hence a high index of suspicion when interpreting imaging is essential. Computed tomography and ventilation perfusion scan can identify bilateral involvement. Some-

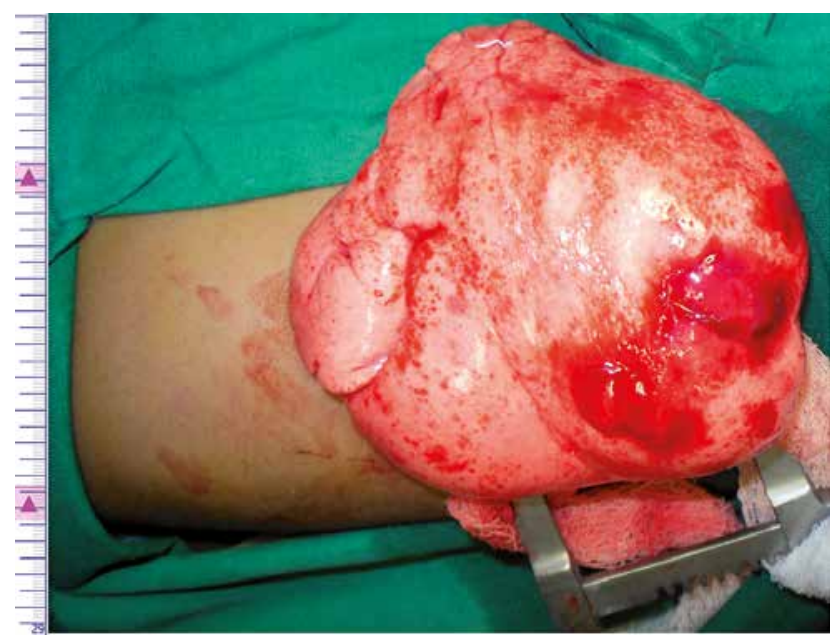

Figure 2. Intra-operative photograph of the massively emphysematous lobe (the attached ruler displays measurements in centimeters)

times, bilateral involvement may settle with only unilateral lobectomy, the distress settling over a period of time. The role of scintigraphy is more relevant in these situations to plan for conservative management [2].

In a child with bilateral CLE, sequential lobectomy offers the best post-operative recovery in terms of pain management, reduction of intra-operative time and avoidance of prolonged anesthesia, bringing down the high risk associated with simultaneous bilateral lobectomy [1, 7].

Outcome is excellent as asymptomatic recovery is the rule in the immediate post-operative period. Long-term follow-up is evident of normalcy with compensatory lung development leading to normal lung functions [1].

In conclusion, the rare occurrence of bilateral CLE in a child, when symptomatic, can be safely and effectively managed by sequential bilateral lobectomy, which is well tolerated with an excellent outcome.

\section{Disclosure}

The author reports no conflict of interest.

\section{References}

1. Demir OF, Hangul M, Kose M. Congenital lobar emphysema: diagnosis and treatment options. Int J Chron Obstruct Pulmon Dis 2019; 14: 921-928.

2. Farrugia MK, Raza SA, Gould S, Lakhoo K. Congenital lung lesions: classification and concordance of radiological appearance and surgical pathology. Pediatr Surg Int 2008; 24: 987-991.

3. Liu YP, Shih SL. Congenital lobar emphysema: appearance on fetal MRI. Pediatr Radiol 2008; 38: 1264.

4. Mei-Zahav M, Konen O, Manson DC, Langer J. Is congenital lobar emphysema a surgical disease? J Pediatr Surg 2006; 41: 1058-1061.

5. Collins LK, Levin TL, Berdon WE, Cowles RA, Newman B. Rudhe syndrome: reversible right middle lobe emphysema in infants with left-to-right shunts: an historical review. Pediatr Radiol 2010; 40: 762-765.

6. Perea L, Blinman T, Piccione J, Laje P. Bilateral congenital lobar emphysema: staged management. J Pediatr Surg 2017; 52: 1442-1445.

7. Shreesha M, Jane RC, Bharat M, Maya D, Dakshesh P. Bilateral congenital lobar emphysema: how should we proceed? Pediatr Surg Int 2005; 21: 659-661. 